\title{
TRANSLATION PLANES OF DIMENSION TWO AND CHARACTERISTIC TWO
}

\author{
N.L. JOHNSON \\ Department of Mathematics \\ University of Iowa \\ Iowa City, Iowa 52242 U.S.A. \\ T.G. OSTROM \\ Department of Pure and Applied Mathematics \\ Washington State University \\ Pullman, Washington 99164 U.S.A. \\ (Received January 4, 1982)
}

ABSTRACT. This article discusses translation planes of dimension two and characteristic two. Let $G$ be a subgroup of the linear translation complement of such a plane $\pi$. The nature of $G$ and its possible action on $\pi$ are investigated. This continues previous work of the authors. It is shown that no new groups occur.

KEY WORDS AND PHRASES. Translation planes, linear translation complement, dimension two and characteristic two.

1980 MATHEMATICS SUBJECT CLASSIFICATION CODE.

\section{INTRODUCTION}

Part I. A translation plane of dimension two over $G F(q)=F$ may be represented by a vector space of dimension four over $F$. The lines through the zero vector form a spread-a class of two-dimensional subspaces which are pairwise independent. The collineations fixing the zero vector (the translation complement) constitute a subgroup of $\Gamma \mathrm{L}(4, q)$; the intersection with $\mathrm{GL}(4, q)$ is the linear translation complement.

Finite translation planes of dimension two have been studied more than those of other dimensions. A complete classification may not be possible if this is understood to include an enumeration of all existing planes. Yet we have reached the stage where we can give a reasonable list of classes when $q$ is even.

Hering [6] has determined the possible groups generated by the elations in the translation complement and one can at least make general statements as to the nature 
of the planes which may admit these groups, especially when the group is nonsolvable.

The authors [8] have investigated the case where the translation complement is not solvable and contains no elations.

In the present paper, $G$ denotes a subgroup of the linear translation complement. We investigate the nature of $G$ and of the planes of dimension two and characteristic two in the following situations:

Part II. $|G|$ is odd.

Part III. All involutions in G are elations, (G may be solvable).

Part IV. All involutions in G are Baer involutions, (G may be solvable).

Part V. G contains elations and Baer involutions. Again no restriction on solvability.

In all of the above, especially in Part V) little attention is paid to the case where $G$ is both solvable and reducible. Some remarks are made about this situation in Part VI.

These results give no recipe for constructing all planes of dimension two and characteristic two. However, there are no new groups and at least some aspects of the possible actions are determined. We suspect that this is as fine a classification as is worthwhile trying to give.

Ostrom's research was supported in part by the National Science Foundation.

2. REDUCIBILITY, $|G|$ ODD.

DEFINITION 2.1. A group $G$ of linear transformation acting irreducibly on a vector space $\mathrm{V}$ is said to be imprimitive if $\mathrm{V}$ is the direct sum of subspaces, to be called subspaces of imprimitivity, such that the image of each subspace of Imprimitivity under each element of $G$ is always a subspace of imprimitivity. Otherwise $G$ is said to be primitive.

DEFINITION 2.2. A group $G$ of linear transformations is said to be fixed-pointfree (f.p.f.) if no non-identity element fixes any non-zero vector. A normal subgroup $G_{1}$ is said to be minimal non f.p.f. with respect to $G$ if $G_{1}$ is not f.p.f. and every normal subgroup of $G$ included in $G_{1}$ is either equal to $G_{1}$ or is f.p.f. 
REMARK. The above two definitions require no restrictions on dimension or characteristic. We wish to remind the reader, however, that throughout this paper $G$ is assumed to be a group of linear translations acting on a vector space of dimension 4 over $G F(q)$, where $q$ is a power of 2 . Indeed $G$ is assumed to be a subgroup of the linear translation complement of a translation plane of dimesion two defined on this vector space.

LEMMA 2.3. Let $G$ be primitive and solvable and let $\mathcal{u}$ be a maximal abelian normal subgroup. Assume that $U$ contains $Z$, where $Z$ is the group of scalars. Then $\boldsymbol{u} \neq \mathrm{Z}$.

PROOF. The proof is by contradiction, so suppose $\mathcal{U}=\mathrm{Z}$ is the unique maximal abelian normal subgroup. Since $G$ is irreducible, $G \neq Z$. If $G$ is solvable $G$ has a normal subgroup $A$ such that $A / A \cap Z$ is abelian and characteristic in $G / Z$. (Let $A$ be the full pre-image of $A / A \cap Z_{.}$)

Suppose that $A$ is f.p.f. Then $A$ must be odd, since no involution is f.p.f. at characteristic 2. An f.p.f. group is a Frobenius complement. See [9] Lemma 2.2. A Frobenius complement which is not cyclic has a characteristic abelian group not in its center. See [10] Theorem 3.7. This contradicts the condition $\mathcal{u}=\mathrm{Z}$. Hence $\mathrm{A}$ is not f.p.f.

Then A contains a subgroup $G_{1}$ which is minimal non-f.p.f. with respect to $G$. Note that $\mathrm{A} / \mathrm{A} \cap \mathrm{Z}$ is abelian implies that $\mathrm{A}$ is nilpotent and hence that the sylow subgroups of $A$ are characteristic. Hence the minimal property of $G_{1}$ implies that $G_{1}$ must be a u=group for some prime $u$. If $H$ is the maximal normal subgroup of $G$ included in $G_{1}$ but not equal to $G_{1}$, then $G_{1} / H$ is elementary abelian. (See [10], Theorem 3.3.)

If $\mathrm{H}$ is trivial, $\mathrm{G}_{1}$ is abelian and by our assumption that $\mathrm{Z}$ is the unique maximal abelian normal subgroup, we could have $G_{1} \subseteq \mathrm{Z}$. This cannot happen since $\mathrm{Z}$ is f.p.f.

Thus $H$ is an f.p.f. u-group. Two-groups are not f.p.f. at characteristic two and the Sylow subgroups of odd order in a Frobenius complement are cyclic. Hence H is abelian and by our present assumeption, $\mathrm{H} \subseteq \mathrm{Z}$.

This implies $u /(q-1)$ and that the minimal $G_{1}$-space have dimension 1 . This last comes from the facts that $q^{2}+1 \equiv 2 \bmod u$ implies that a $u$-group fixes at least two 
components of the spread and $q+1 \equiv 2 \bmod u$ implies that a fixed 2-space must include at least two fixed 1-spreads. Cliffords Theorem (see [3] page 70) implies that if $G$ is primitive, all of the minimal $G_{1}$-spaces must be isomorphic as $G_{1}$-modules and hence that $G_{1}$ must be faithful on its minimal $G_{1}$-spaces. Since this does not happen, we have a contradiction to the assumption that $\mathscr{Q}=\mathrm{Z}$.

THEOREM 2.4. Let $G$ be primitive and solvable. Then $G$ is isomorphic to a subgroup of $\Gamma L\left(1, q^{4}\right)$.

PROOF. Let $\mathscr{U}$ be a maximal abelian normal subgroup of $G$ such that $\mathscr{U}$ contains Z. By the previous Lemma, $थ \neq \mathrm{Z}$.

If $\mathcal{U}$ is not f.p.f., it leaves invariant the subspace pointwise fixed by one of its elements and hence is not faithful on its minimal subspaces. Thus Cliffords Theorem implies that $\mathcal{U}$ is f.p.f.

Then (see Hering [3] Hilfsatz 5) $G$ is isomorphic to a subgroup of $\Gamma L\left(1, q^{4}\right)$ or $\Gamma L\left(2, q^{2}\right)$ since $G$ is primitive.

If $G \tilde{c} \Gamma L\left(1, q^{4}\right)$, we are done, so suppose $G$ is a subgroup of $\Gamma L\left(2, q^{2}\right)$. We may also assume $G \cap \mathrm{GL}\left(2, \mathrm{q}^{2}\right)$ centralizes 2 .

$G>G \cap \operatorname{SL}\left(2, q^{2}\right)$. The solvable subgroups of $\operatorname{SL}\left(2, q^{2}\right)=\operatorname{PSL}\left(2, q^{2}\right)$ (for $q$ even) are cyclic or dihedral except in cases where there is a characteristic 2-group. G has no normal 2-group if it is primitive so $G \cap S L\left(2, q^{2}\right)$ is cyclic or dihedral and hence contains a characteristic abelian group $U_{1}$. Since $U$ is maximal and $U$ is centralized by $U_{1}$, $2 U_{1}$ is abelian so $U_{1} \subseteq U$. But $U$ is actually included in the center of $\mathrm{GL}\left(2, \mathrm{q}^{2}\right)$, so $\mathcal{U} \cap \mathrm{SL}\left(2, \mathrm{q}^{2}\right)$ is trivial. Hence $\mathrm{G} \cap \mathrm{SL}\left(2, \mathrm{q}^{2}\right)$ is trivial. At characteristic 2. $\mathrm{GL}\left(2, \mathrm{q}^{2}\right)$ is the direct product of $\operatorname{SL}\left(2, \mathrm{q}^{2}\right)$ and the center of $\mathrm{GL}\left(2, q^{2}\right)$. Hence, in our case, $G \cap \mathrm{GL}\left(2, q^{2}\right)=\mathcal{u}$. In that case $G=G \cap \Gamma L\left(2, q^{2}\right)$ cannot act irreducibly on the vector space of dimension 4 over GF(q). Thus the only possibility left is $G$ is isomorphic to a subgroup of $\Gamma L\left(1, q^{4}\right)$.

THEOREM 2.5. If $G$ is irreducible but imprimitive then $G$ has a reducible suggroup of index 2 .

PROOF. The theorem is trivial if the vector space is the direct sum of two subspaces of imprimitivity. 
Hence assume that the basic vector space $V$ is the direct sum $V=V_{1} \oplus V_{2} \oplus V_{3} \oplus V_{4}$ of subspaces of imprimitivity of dimension 1 . Let $G_{0}$ be the normal subgroup of $G$ which fixes each of $v_{1}, v_{2}, v_{3}, v_{4}$.

By Clifford's Theorem, $V$ is a direct sum $W_{1} \oplus \cdots \oplus \mathrm{W}_{k}$ of invariant $G_{0}$ spaces; the minimal $G_{0}$-spaces in $W_{i}$ for fixed $i$ are all isomorphic on $G_{0}$-modules and each minimal $G_{0}$-space belongs to one of the $W_{i}$ 's; the $W_{i}$ all have the same dimension. Note that $k=1,2$, or 4 . If $k=2$ we are done.

If $k=4$ then there are no minimal $G_{0}$-spaces besides $v_{1}, v_{2}, V_{3}, v_{4} \cdot\left|G_{0}\right|$ cannot be even, since an involution in $G_{0}$ would have to fix $v_{1}, v_{2}, v_{3}, v_{4}$ pointwise.

If $G_{0}$ is odd, then $G_{0}$ must fix some other 1-space on the component of the spread which contains $v_{1}$. We conclude that if $k=4$, there must be two components, each containing two of the $V_{i}$. In this case $G$ has a subgroup of index two fixing these two components.

We have left the case where $k=1$, so that all of the minimal spaces are 1somorphic as $G_{0}$-modules. We may assume that $G$ and $G_{0}$ contain all of the scalars. (If they do not, adjoin the scalars.) If the minimal $G_{0}-$ spaces are isomorphic as $G_{0}$ modules, then $G_{0}$ must be faithful on its minimal subspaces. With the minimal subspaces being 1-dimensional, it follows that if $G_{0}$ contains the scalars 2 , then $G_{0}=Z$.

But $G / G_{0}$ is a subgroup of the symmetric group $S_{4}$ and its order is divisible by 4. $\left|S_{4}\right|=24$ and any subgroup whose order is divisible by 4 is one of the following: (a) a two-group, (b) $\mathrm{A}_{4}$, (c) $\mathrm{S}_{4}$. In each case there is a normal 2-group (not necessarily a Sylow 2-group).

Let $\bar{G}=G / Z=G / G_{0}$. Let $S$ be a 2 -group in $G$, where $\bar{S}$ is normal in $\bar{G}$. If $\sigma$ is an involution in $S$ and $\lambda \in G$ then $\lambda^{-1} \sigma \lambda=\sigma_{1} \nu$ for some $\sigma_{1}$ in $S$ and some $\nu \in Z$. Hence $1=\left(\sigma_{1} \nu\right)^{2}=\sigma_{1}{ }^{2} \nu^{2}=\nu^{2}$ and $\nu=1$. Then the subgroup of $\mathrm{S}$ generated by its involutions is normal in $G$. Hence the subspace which is pointwise fixed by this 2-group is invariant under $G$ and $G$ is reducible, contrary to hypothesis. Hence the case $k=1$ does not happen and the Theorem is proved.

THEOREM 2.6. Suppose that $Q(G)$ is irreducible. Then $G \tilde{\subseteq} \Gamma L\left(1, q^{4}\right)$. PROOF. If $\theta(G)$ is irreducible, it may be primitive by the previous Theorem. Then $\theta(G) \cong\left[L\left(1, q^{4}\right)\right.$ by (2.4). Since $G$ must preserve 1-spaces over $G F(1)$, 
$\theta(G) \cap \mathrm{GL}\left(1, q^{4}\right)$ is a subgroup of $\theta(G)=\theta(G) \cap \Gamma L\left(1, q^{4}\right)$ whose index divides 4 . But $|\theta(G)|$ is odd, so $\theta(G) \simeq G L\left(1, q^{4}\right)$ and $\theta(G)$ is cyclic. The theorem then follows from Proposition 19.8 in Passman [13].

THEOREM 2.7. Suppose that $G=\theta(G)$ contains the scalars and that $G$ is reducible. Then $G$ is cyclic or $G$ is not faithful on one of its minimal subspaces.

PROOF. If $G$ is reducible, it has some invariant 2-space $V_{1}$. If $G$ is not faithful on $V_{1}$ we are done. Otherwise $G$ is a subgroup of $G L(2, q)$. The subgroups of $\operatorname{PSL}(2, q), q=2^{r}$ of odd order are cyclic. See Dickson [1]. But $\operatorname{PSL}(2, q)=\operatorname{SL}(2, q)$. Thus $\mathrm{G} \cap \operatorname{SL}(2, q)$ is cyclic, with order dividing $q-1$ or $q+1$. As a subgroup of GL $(2, q)$ acting on a vector space of dimension two, $G$ must either be cyclic or have a pair of invariant 1-spaces and not be faithful on at least one of them.

3. G GENERATED BY ELATIONS

In this section we are concerned with the case where the translation complement contains elations. Actually, we will limit ourselves to the case where $G$ is the subgroup of the translation complement generated by the elations.

Hering 6 has investigated this situation. In one of his cases, $G$ has a normal subgroup of index two and odd order. In unpublished work he has shown that, under much broader circumstances than we have here, G must be dihedral. Since this work is not published, we will have a proof that $G$ is dihedral for a plane of dimension two over $\mathrm{GF}(\mathrm{q})$, where $\mathrm{q}$ is a power of 2 .

LEMMA 3.1. Suppose that (a) G has a normal subgroup of index and odd order; (b) G is primitive; (c) The involutions in G are elations; (d) G contains more than one elation. Then $G$ is dihedral.

PROOF. Let $\sigma$ be an involution and $\tau$ an element of odd order such that $\langle\tau\rangle$ has index 2 in $\langle\sigma, \tau\rangle$. Then the involutions have the form $\sigma \tau^{i}$ for those $i$ such that $\sigma \tau^{i} \sigma=\tau^{-i}$. Hence the group generated by the involutions is dihedral.

By (2.4) $G$ is isomorphic to a subgroup of $\Gamma L\left(1, q^{4}\right)$. Then $G \cap G L\left(1, q^{4}\right)$ is a cyclic normal subgroup of odd order. The fact that $G$ is a group of linear transformations over $\mathrm{GF}(\mathrm{q})$ implies that the index of $\mathrm{G} \cap \mathrm{GL}\left(1, \mathrm{q}^{4}\right)$ in $\mathrm{G}$ must divide 4 . Because of (a) this index is 2 . Hence (d) and the above argument implies that $G$ is dihedral since it is generated by its elations. 
LEMMA 3.2. $G$ is dihedral if $G$ is reducible and the conditions other than (b) of (3.1) are satisfied.

PROOF. If there are two elations with the same axis, 4 divides $|G|$. If there are two elations with different axes, no component of the spread is invariant. If $G$ has an invariant 1-space, the component containing this 1-space is invariant. Hence if $G$ is reducible, it has an invariant subspace which is a Baer subplane $\pi_{0}$.

If $G$ is faithful on $\pi_{0}$, the hypotheses are sufficient to guarantee that $G$ is dihedral.

Otherwise, let $G_{1}$ be a maximal dihedral subgroup of $G$ acting faithfully on $\pi_{0}$. Specifically, let $G_{1}=\langle\sigma, \tau\rangle$ where $\rho$ is an involution and $\langle\tau\rangle$ is the cyclic stem. If $G$ is not dihedral, there is some involution $\sigma$ such that $\left\langle\sigma, G_{1}\right\rangle$ is not dihedral and some element fixes $\pi_{0}$ pointwise.

Suppose that $\sigma \rho \tau^{i}$ fixes $\pi_{0}$ pointwise. Then the restrictions to $\pi_{0}$ of the two involutions $\sigma$ and $\rho \tau^{i}$ are identical. In particulat, they fix the same points of $\pi_{0}$ so that the axes of the elations $\sigma$ and $\rho \tau^{i}$ must be the same. But this requires that either $\sigma=\rho \tau^{i}$ or that $G$ has a subgroup of order 4 .

Neither of these conditions is satisfied. Suppose that $\sigma \tau^{i}$ fixes $\pi_{0}$ pointwise. Then the restrictions of $\sigma$ and $\tau^{-i}$ to $\pi_{0}$ are identical, so $\tau^{-2 i}$ must fix $\pi_{0}$ pointwise. But $G_{1}=\langle\rho, \tau\rangle$ is faithful on $\pi_{0}$ so $i=0$. In this case, $\sigma$ fixes $\pi_{0}$ pointwise, contrary to the assumptions that $\sigma$ is an elation.

LEMMA 3.3. Suppose that $\mathrm{G}$ is irreducible but imprimitive and the conditions of (3.1) excluding (b) are satisfied. Then $\mathrm{G}$ is dihedral.

PROOF. By (2.5) G has a reducible subgroup $H$ of index 2 . We may assume that $\mathrm{V}=\mathrm{V}_{1} \oplus \mathrm{V}_{2}$, where $\mathrm{V}_{1}$ and $\mathrm{V}_{2}$ have dimension 2, are interchanged by each involution $\mathrm{G}$ and some invariant under $\mathrm{H}$.

Lemmas (2.3), (2.4), (2.5), (2.6) for a translation plane of dimension 2 over its kernel and a vector space of dimension 4 over GF(q) apply as well as to a vector space of dimension two, i.e., to a Desarguesian affine plane over GF(q).

Hence if $H$ is irreducible and faithful on $V_{1}$ and has odd order, then $H$ is cyclic as in $(2.6)$. 
If $H$ is faithful on $V_{1}$ and is reducible on $v_{1}$ then $i t$ has at least two invariant 1-spaces and is a subgroup of the direct product of two cyclic groups. Hence $\mathrm{H}$ is abelian. If $H$ is f.p.f. and abelian, then $H$ is cyclic.

Since $H$ has two fixes 1 -spaces in $V_{2}, V$ must be the direct sum of 4 H-invariant 1-spaces. If $\mathrm{H}$ has an additional 1-space in $\mathrm{V}_{1}\left(\right.$ or $\left.\mathrm{V}_{2}\right)$ then $\mathrm{H}$ fixes each 1-space in $v_{1}\left(V_{2}\right)$. If $\mathrm{H}$ is not cyclic, it is not f.p.f. on $v_{1}$. Thus if $H$ fixes each 1-space in $v_{1}$, then $H$ is not faithful on $v_{1}$.

If $H$ has precisely 2 invariant 1-spaces in $v_{1}$ and precisely 2 invariant 1-spaces in $v_{2}$ then therefore 1-spaces must occur in two orbits of length 2 under $G$. In this case, the 2-space containing an invariant pair is invariant so $G$ is reeucible and we can return to the previous case.

We have left the case where $H$ is not faithful on $v_{1}$ or $v_{2}$.

Let $G_{1}=\langle\rho, \tau\rangle$ be a maximal (dihedral) subgroup where $\langle\tau\rangle \subseteq H$ acts faithfully on $V_{1}$ and $\rho$ is an involution. If $G \neq G_{1}$ and $G$ is generated by its involutions, there is an involution $\sigma$ such that $\left\langle\sigma, G_{1}\right\rangle$ contains an element which fixes $v_{1}$ pointwise. Note that $v_{1}$ is not invariant under any element of the form $\sigma \tau^{1}$ or $\rho \tau^{1}$. If $\sigma \rho \tau^{i}$ fixes $v_{1}$ pointwise, we get a contradiction just as in the proof of the previous Lemma. We conclude that $G$ must be dihedral.

THEOREM 3.4. Let $G$ be a subgroup of the linear translation complement of a translation plane of dimension 2, characteristic 2. Suppose that $G$ is generated by its elations. Then one of the following holds:

(a) G is a Suzuki group.

(b) $\mathrm{G} \cong \mathrm{SL}\left(2,2^{\mathrm{s}}\right)$ for some $\mathrm{s}$.

(c) G is elementary abelian and is a group of elations all with the same axis.

(d) $G$ is dihedral.

PROOF. This is just a restatement of a Theorem of Hering [6, 3] except for case (d). In Hering's remaining case, $G$ has a subgroup of odd order and index 2. If $|H|$ is odd and $[G: H]=2$ then $G$ is dihedral so we get case (d) by applying (3.1), (3.2), and (3.3).

REMARK. By Hering [7], Theorem (5.1) in case (b) of (3.4), G has an invariant subplane which is a Lüneburg plane. 


\section{ALL INVOLUTIONS ARE BAER INVOLUTIONS}

Here we are concerned with a subgroup of the linear translation complement which has even order but contains no elations. As pointed out in [8], the Sylow 2-groups are elementary abelian. The case where $G$ is non-solvable is covered in [8], so we shall be mostly concerned with the solvable case.

LEMMA 4.1. Let $G$ be a subgroup of the linear translation complement of a finite translation plane of even square order. Suppose that each Sylow 2-group fixes a Baer subplane pointwise and that there is more than one Sylow 2-group. Then no Sylow 2-group contains a non-trivial element which normalizes another Sylow 2-group.

PROOF. Suppose that $S_{1}$ and $S_{2}$ are Sylow 2-groups and that $s_{1}$ contains an involution $\rho$ which normalizes $S_{2}$. Then $\rho$ centralizes some involution $\sigma$ in $S_{2}$. Hence $\langle\rho, \sigma\rangle$ is a group of order 4 contained in some sylow 2-group $\mathrm{S}_{3}$.

Then the Baer subplanes pointwise fixed by $\sigma, \rho, s_{1}, s_{2}, s_{3}$ must all be identical. By Foulser [2], Theorem 3, a Sylow 2-group of the pointwise stablizer of a Baer subplane is normal and hence unique (in the pointwise stabilizer). Hence $S_{1}$ and $\mathrm{S}_{2}$ are not distinct.

LEMMA 4.2. If $G$ is solvable and each Sylow 2-group fixes a Baer subplane pointwise, then either the Sylow 2-group is normal or $|G|$ is not divisible by 4.

PROOF. See Hering [5]. Theorem 1. If $S$ is an elementary abelian 2-group and is a Frobenius complement then $|S|=2$.

REMARK. Lemma (4.1) does not require that the plane have dimension 2 over its kerne1. Foulser's Theorem 3 [2] implies that the Sylow 2-group must be elementary abelian in this case. Hering's Theorem 1 then implies that if $G$ is non-solvable and generated by its Sylow 2 -group it must be isomorphic to $\mathrm{SL}\left(2,2^{s}\right)$ for some $s$.

LEMMA 4.3. If $G$ contains no elations, the Sylow 2-groups are elementary abelian. If, in addition, $G$ is solvable and generated by its involutions, then $G / O(G)$ is a Sylow 2-group.

PROOF. A Sylow 2-group must have at least one fixed component. If it contains no elations, it acts faithfully on a 2-space and is a subgroup of a Sylow 2-group of GL $(2, q)$. Hence the Sylow 2-groups are elementary abelian. 
The last part follows from Walter's characterization of groups with abelian Sylow 2-groups [16].

LEMMA 4.4. If $\mathrm{G}$ is irreducible and solvable, the Sylow 2-groups have order 2 or 4. In the latter case $G$ interchanges a pair of two dimensional subspaces and the collineation group induced on them by their stablizer is dihedral.

PROOF. If $G$ is primitive, then $G \subseteq \Gamma\left(1, q^{4}\right)$ by $(2.4)$. But $G$ is acting as a group of linear transformations over $G F(q)$. When $G$ is represented as a subgroup of $\Gamma L\left(1, q^{4}\right)$, the semi-linear transformations over $G F\left(q^{4}\right)$ must be those involving automorphisms of $\operatorname{GF}\left(\mathrm{q}^{4}\right)$ which fix the elements of $\mathrm{GF}(\mathrm{q})$. The multiplicative group of GF $\left(q^{4}\right)$ is odd-i.e., GL $\left(1, q^{4}\right)$ has odd order. Thus the Sylow 2-groups are cyclic. By (4.3), they are elementary abelian. Hence the Lemma is proved for the case where $G$ is primitive, since the Sylow 2-groups must have order 2 .

By (2.5) if $G$ is irreducible but imprimitive then $G$ has a reducible subgroup $G_{0}$ of index $w$ and $G_{0}$ has a pair of invariant 2-spaces $V_{1}$ and $V_{2}$. The subgroup fixing $v_{1}$ (or $v_{2}$ ) pointwise must have odd order since an involution fixing $V_{1}$ pointwise would have to act f.p.f. on $V_{2}$ and an involution cannot act f.p.f. on an invariant space at characteristic two.

Suppose that $G_{0}$ has a subgroup of order 4 fixing pointwise a 1-space in $V_{1}$. (It will also fix pointwise a subspace of $v_{2} \cdot$ ) If this subspace is invariant under $G_{0}$, then $G_{0}$ will have a characteristic 2-group; the subspace fixed pointwise by this subgroup will be invariant under $G$ so $G$ is reducible, contrary to hypotheses.

Otherwise $G_{0}$ must induce a dihedral group on $V_{1}$, since $G_{0}$ is solvable. Hence the Sylow 2-groups in $G_{0}$ have order 2; those in $G$ have order 4.

LEMMA 4.5. Suppose that $G$ is reducible and solvable and that the Sylow 2-groups have order $>2$. Then $G$ has an invariant subspace pointwise fixed by a $2-$ group. If $G$ is not a 2-group, either it contains a group of affine homologies or its minimal invariant subspaces have dimension 2 and the induced group is dihedral.

PROOF. If $G$ is reducible, it has an invariant 2-space since if its minimal space is 1-dimensional, G must fix the component which contains this 1-space.

Let $V_{1}$ be an invariant 2-dimensional subspace. If the subgroup fixing $V_{1}$ pointwise has even order, we are done. 
If $\mathrm{H}$ is the subgroup fixing $\mathrm{V}_{1}$ pointwise and $|\mathrm{H}|$ is odd, consider the induced group $G / V_{1}$. If the $2-$ group in $G / V_{1}$ has order greater than 2 , then $G / V_{1}$ has an invariant subspace in $V_{1}$, since $G$ is assumed to be solvable. This subspace, as a vector space in the whole plane, will be invariant under $G$ and pointwise fixed by a Sylow 2-group.

If a minimal G-space is 1-dimensional and $G$ is not a 2 -group then $G$ cannot act faithfully on the component which contains this 1-space. Hence G contains a group of homologies. (In this section G contains no elations.)

If the minimal G-spaces have dimension 2 and $G$ is not a 2-group but is solvable, the induced group must be dihedral.

THEOREM 4.6. Let $\mathrm{G}$ be a nontrivial subgroup of the linear translation complement for a plane $\pi$ of dimension 2 over $G F(q)$, where $q$ is even. Suppose that $G$ is generated by its involutions and they are all Baer involutions. Then at least one of the following holds:

(a) $\quad G=S L\left(2,2^{s}\right)$ for some power of $s, \pi$ has an Ott subplane of order $2^{s}$ on which $G$ acts in the normal manner. G acts irreducibly on $\pi$.

(b) $\mathrm{G}=\mathrm{SL}\left(2,2^{\mathrm{S}}\right)$. $\mathrm{G}$ is reducible. Each Sylow 2-group fixes a Baer subplane pointwise. All of these Baer subplanes are included in a derivable net. In the derived plane all of the involutions are elations.

(c) $\theta(G)$ has index 2 in $G$ and $G$ is dihedral.

(d) The Sylow 2-groups in G have order 4. G interchanges a pair of 2-dimensional subspaces and the group induced on them be their stabilizer is dihedral.

(e) G is reducible and one of its invariant subspaces is pointwise fixed by a 2-group. If $\mathrm{G}$ is not a 2-group either it contains affine homologies or the minimal $G$ spaces have dimension 2 and $G$ induces a dihedral group on its minimal space.

PROOF. If $G$ is non-solvable, we have (a) or (b) by [8]. If $G$ is solvable and each Sylow 2-group fixes a Baer subplane pointwise apply (4.2). In the remaining cases, apply (4.4) and (4.5). 


\section{GROUPS GENERATED BY INVOLUTIONS}

In the last two sections, we have assumed that the involutions in the linear translation complement are all of the same kind-i.e., either they are all elations or all Baer involutions. As before $G$ always denotes a subgroup of the linear translation complement.

LEMMA 5.1. If $G$ contains both elations and Baer involutions then the group generated by the Baer involutions contains elations.

PROOF. If $G$ contains involutions of both kinds, let $G_{1}$ be the normal subgroup generated by all of the elations in $G$ and let $G_{2}$ be the normal subgroup generated by all of the Baer involutions in $G$.

Let $S$ be a Sylow 2-group and let $S_{1}=S \cap G_{1}, S_{2}=S \cap G_{2}$. By Hering's results [6], $G_{1}$ contaions no Baer involutions. If $G_{2}$ contains no elations, then $S_{1} S_{2}=S_{1} \times S_{2}$. Furthermore $S_{1}$ and $S_{2}$ are both elementary abelian. See (4.3) and again refer to Hering [6]. It follows that $\mathrm{s}_{1} \times \mathrm{s}_{2}$ is elementary abelian and contains involutions which do not belong to either $\mathrm{s}_{1}$ or $\mathrm{s}_{2}$ (unless one of them is trivial). But every involution belongs to $G_{1}$ or $G_{2}$ so every involution in $S$ belongs to $S_{1}$ or $S_{2}$. Hence $\mathrm{G}_{2}$ must contain elations.

DEFINITION 5.2. In the rest of this section $G_{1}$ is the group generated by the elations; $C\left(G_{1}\right)$ is the centralizer of $G_{1}$ in $G$ and $H$ is the subgroup of $C\left(G_{1}\right)$ generated by the involutions.

LEMMA 5.3. The center of $G_{1}$ is trivial unless $G_{1}$ is a 2-group. Thus $G_{1} C\left(G_{1}\right)=G_{1} \times C\left(G_{1}\right)$

PROOF. By (3.4) $G_{1}$ is a Suzuki group, $S L\left(2,2^{s}\right)$, dihedral, or a 2-group. The two non-solvable cases are simple. A dihedral group with cyclic stem of odd order has a trivial center.

LEMMA 5.4. Suppose that $G_{1}$ is not trivial, that $C\left(G_{1}\right)$ contains involutions and neither $G_{1}$ nor $C\left(G_{1}\right)$ has a non-trivial normal 2-group. Then the axes of the elations in $G_{1}$ can be embedded in a derivable net which contaions the Baer subplanes pointwise fixed by involutions in $C\left(G_{1}\right)$. If $C\left(G_{1}\right)$ is non-solvable, this derivable net is embedded in the given translation plane. 
PROOF. If $C\left(G_{1}\right)$ is non-solvable this follows from (2.7) in [8], since $C\left(G_{1}\right)$ must be reducib]e. Each axis of an elation in $G_{1}$ is invariant under $C\left(G_{1}\right)$. If $G_{1}$ has no normal 2-group, there are at least three such axes.. Hence the non f.p.f. elements in $C\left(G_{1}\right)$, including the involutions, must fix Baer subplanes pointwise. The Lemma is now a direct consequence of the classical theory concerning a regular determined by three skew projective lines, etc.

In the rest of this section, the subgroup $G_{1}$ is assumed to be non-trivial. LEMMA 5.5. If $G_{1}$ is not a 2-group, $C\left(G_{1}\right)$ is isomorphic to a subgroup of $\operatorname{GL}(2, q)$.

PROOF. By (5.2), $G_{1} \cap C\left(G_{1}\right)$ is trivial, so $C\left(G_{1}\right)$ contains no homologies. If $C\left(G_{1}\right)$ contains an affine homology whose axis $\ell$ is the axis of an elation in $G_{1}$ then $\ell$ must be invariant under $G_{1}$ and all of the elations in $G_{1}$ fix $\ell$. Hence $G_{1}$ is a 2-group, contrary to hypotheses.

Thus, $C\left(G_{1}\right)$ must be faithful on each elation axis. This implies that $\left(G_{1}\right) \simeq \mathrm{GL}(2, q)$

LEMMA 5.6. If $G_{1}$ is not a two-group, $G / G_{1} C\left(G_{1}\right)$ is abelian. If $H$ is not a 2-group (or trivial) G/H C (H) is abelian.

PROOF. If $\lambda \in G$, the mapping $G_{1} \rightarrow \lambda^{-1} G_{1} \lambda$ is an inner automorphism of $G_{1}$ if and only if belongs to $G_{1} C\left(G_{1}\right)$. Hence $G / G_{1} C\left(G_{1}\right)$ is isomorphic to a subgroup of the group of outer automorphisms of $G_{1}$. By (3.4), $G_{1}$ is dihedral, a Suzuki group, or $\mathrm{SL}\left(2,2^{\mathrm{s}}\right)$ for some $\mathrm{s}$.

If $G_{1}$ is dihedral, the cyclic stem has odd order. Let $G_{1}=\langle\rho, \tau\rangle$ where $\rho$ is an involution and $\langle\tau\rangle$ is the cyclic stem. All of the involutions in $G_{1}$ are equivalent under the inner automorphisms so the outer automorphisms fix $\rho$ and induce automorphisms of $\langle\tau\rangle$. The automorphism group of a cyclic group is abelian, so the automorphism group of $\mathrm{G}_{1}$ is abelian in this case.

The outer automorphisms of $\mathrm{SL}\left(2,2^{5}\right)$ are essentially the field automorphisms; this group is cyclic.

According to Suzuki [15], Theorem 11, page 139, the same remark holds for the 
$H$ is normal in $G$; if $H$ is not a 2 -group then $H$ is dihedral or $\operatorname{SL}\left(2,2^{5}\right)$ for some $s$ since $H$ is a subgroup of $G L(2, q)$ generated by involutions. (Note that $H \cap G_{1}$ must be trivial unless $H$ and $G_{1}$ are both 2-groups.) Thus the arguments just used also apply to $\mathrm{H}$.

THEOREM 5.7. Except in the cases where $H$ is trivial and $G_{1}$ is dihedral or where $G$ has a normal 2-group, $G_{1} C\left(G_{1}\right)$ contains the group generated by the involutions in $G$ and if $G_{1}$ is not a Suzuki group $G=G_{1} C\left(G_{1}\right)$.

PROOF. As in the proof of Lemma (5.6), $G / G_{1} C\left(G_{1}\right)$ is isomorphic to a group of outer automorphisms of $G_{1}$.

Thus the first part of the Theorem is true unless $G$ contains some involution not in $G_{1} C\left(G_{1}\right)$ which induces an outer automorphism of $G_{1}$.

If $G_{1}=S z\left(2^{S}\right)$ then $s$ must be odd. The Theorem of Suzuki referred to before implies that the outer automorphism group has odd order.

If $G_{1}=\operatorname{SL}\left(2,2^{s}\right)$ then the net whose components are elation axes is Desarguesian. This net is invariant under G.

By Ostrom [11], the induced permutation group on the affine elation centers is isomorphic to a subgroup of $\operatorname{P\Gamma L}\left(2,2^{s}\right)$ for some $s$.

If $\left|G_{1}\right|$ and $\left|C\left(G_{1}\right)\right|$ are both even we can apply (5.4) to infer that the elation axes are embedded in a regular (derivable) net invariant under $\dot{G}$. (We do not need the condition that all components of this derivable net are components of the spread which defines the plane.) Again the induced group on the affine elation centers is a subgroup of $\operatorname{P\Gamma L}\left(2,2^{s}\right)$. An element of $G$ fixes all of the elation centers if and only if it is in $C\left(G_{1}\right)$. Thus if $G_{1}$ is not a Suzuki group $G / C\left(G_{1}\right)$ is a subgroup of $\operatorname{P\Gamma L}\left(2,2^{s}\right)$. Let $\widetilde{G}=G / C\left(G_{1}\right)$.

Furthermore if $G_{1}=\operatorname{SL}\left(2,2^{s}\right), G F\left(2^{s}\right)$ must be a subfield of $G F\left(q^{2}\right)$. If $2^{s}=q$ the plane must be Desarguesian. See Prohaska [14]. If the plane is not Desarguesian, $G F\left(2^{s}\right)$ will be a proper subfield of $G F(q)$. This will also be the case where $H$ is nontrivial and $G_{1}$ is dihedral by (5.4). Since we are looking at linear transformations over GF(q), it follows that an element of $G$ which fixes three elation centers must fix all of them and thus be in $C\left(G_{1}\right)$. Hence $\widetilde{G}$ is in $\operatorname{PGL}\left(2,2^{s}\right)=\operatorname{PSL}\left(2,2^{s}\right)$, not merely in $\operatorname{P\Gamma L}\left(2,2^{\mathrm{S}}\right)$. 
If $\widetilde{G}$ should have a normal 2-group, so would $G$. Recall that $G_{1} \cap C\left(G_{1}\right)$ is trivial, so $\widetilde{G}$ has a normal subgroup isomorphic to $G_{1}$. From Dickson's [1] 1ist of subgroups of $\operatorname{PSL}\left(2,2^{s}\right), \widetilde{G}$ is dihedral or $\operatorname{PSL}\left(2,2^{t}\right)$ for some $t$. Hence if $G_{1}$ is $\mathrm{SL}\left(2,2^{8}\right), \widetilde{G}$ is $\operatorname{SL}\left(2,2^{5}\right)$; if $G_{1}$ is dihedral, $\widetilde{G}$ is dihedral and the cyclic stem must have odd order since $\widetilde{G}$ can have no normal 2 -group. Hence the involutions in $\widetilde{G}$ are all conjugate. Hence they must all be in $G_{1}$. Hence $\widetilde{G}=G_{1}$.

COROLLARY 5.8. If the group generated by the elations is A Suzuki group, G contains no Baer involutions.

PROOF. By (5.7) $G=G_{1} C\left(G_{1}\right)=G_{1} \times C\left(G_{1}\right)$ where $G_{1}$ is, in this case a Suzuki group. If $G$ contains Baer involutions, then $C\left(G_{1}\right)$ will contain Baer involutions. This implies that $G_{1}$ induces a Suzuki group on a Desarguesian plane of order $q$; that is, on a Baer subplane pointwise fixed by some element of $C\left(G_{1}\right)$.

LEMMA 5.9. Suppose that $\pi$ has a derivable net $\eta_{1}$. Let $\eta_{2}$ be the net whose components are the Baer subplanes in $n_{1} \cdot\left(n_{1}\right.$ and $n_{2}$ correspond to a regulus and its opposite regulus.) Then $G$ cannot contain 2-group $Q_{1}$ and $Q_{2}$ with the following properties:

(a) $Q_{1}$ and $Q_{2}$ centralize each other.

(b) $\eta_{1}$ and $\eta_{2}$ are invariant under $Q_{1} Q_{2}$.

$Q_{1}$ consists of elations with a given axis $l$ in $\eta_{1} ; Q_{2}$ fixes pointwise some Baer subplane $V\left(Q_{2}\right)$ of $\eta_{1}$ (line of $\left.\eta_{2}\right)$.

(c) $Q_{1}=Q_{2}=q_{0}>2 . Q_{1}$ has an invariant subplane of order $q_{0}$ embedded in $\mathrm{V}\left(\mathrm{Q}_{2}\right)$. In its action on $\eta_{2}, Q_{2}$ has an invariant subplane of order $q_{0}$ embedded in $l$, where $\ell$ now is a Baer subplane of $\eta_{2}$.

We can choose a representation so that the components of $\eta_{1}$ are $\mathrm{x}=0$ and $\mathrm{y}=\mathrm{xW}$, where W runs over the various scalar matrices over GF(q). Furthermore, we can choose a basis so that $Q_{1}$ is represented by the various matrices of the form

$$
\left(\begin{array}{llll}
1 & 0 & B & 0 \\
0 & 1 & 0 & B \\
0 & 0 & 1 & 0 \\
0 & 0 & 0 & 1
\end{array}\right)
$$

$\beta \in \operatorname{GF}\left(q_{0}\right)$ and $v\left(Q_{2}\right)$ is the set of points $\left(x_{1}, x_{2}, y_{1}, y_{2}\right)$ such that $x_{2}=y_{2}=0$. This representation for $Q_{1}$ is unchanged under conjugation with respect to any matrix 
$\left(\begin{array}{ll}A & \theta \\ \theta & A\end{array}\right)$ where $A$ is 2 by 2 over $G F(q)$.

Hence $Q_{2}$ can be represented by matrices of the form

$$
\left(\begin{array}{llll}
1 & \alpha & 0 & 0 \\
0 & 1 & 0 & 0 \\
0 & 0 & 1 & \alpha \\
0 & 0 & 0 & 1
\end{array}\right)
$$

where varies over $\mathrm{GF}\left(\mathrm{q}_{0}\right)$.

Let $m=\{M \mid y=x M$ is a component of $\pi\}$. Note that if $M \in M$ then $M+I B$ and $\left(\begin{array}{ll}1 & \alpha \\ 0 & 1\end{array}\right)$ M $\left(\begin{array}{ll}1 & \alpha \\ 0 & 1\end{array}\right) \quad$ both belong to $m$. Let $M=\left(\begin{array}{ll}m_{1} & m_{2} \\ m_{3} & m_{4}\end{array}\right)$ and suppose that $\mathrm{m}_{3} \in \mathrm{GF}\left(\mathrm{q}_{0}\right)$. Then

$$
\left(\begin{array}{ll}
1 & \alpha \\
0 & 1
\end{array}\right)\left(\begin{array}{ll}
m_{1} & m_{2} \\
m_{3} & m_{4}
\end{array}\right)\left(\begin{array}{ll}
1 & \alpha \\
0 & 1
\end{array}\right)=\left(\begin{array}{c}
m_{1}+\alpha m_{3} \\
m_{3}
\end{array}\right)\left(\begin{array}{c}
m_{1}+\alpha m_{3}+m_{2}+\alpha m_{4} \\
m_{3} \alpha+m_{4}
\end{array}\right)
$$

and $M+\alpha m_{3} I=\left(\begin{array}{c}m_{1}+\alpha m_{3} \\ m_{3}\end{array}\right)\left(\begin{array}{c}m_{2} \\ m_{3} \alpha+m_{4}\end{array}\right)$.

But the difference of distinct matrices in $m$ must be non-singular. Thus if matrices in $m$ have identical first columns (second rows) they must be identical. Hence $m_{2}=m_{1}+2 m_{3}+m_{2}+m_{4}$ for all $\alpha$ in $G F\left(q_{0}\right)$. Thus $\alpha\left[\left(m_{1}+m_{4}\right)+\alpha m_{3}\right]=0$. This can only happen if $m_{3}=0, m_{1}=4$. But if the $q^{2}$ distinct matrices in $m$ have distinct second rows every ordered pair of elements of GF(q) must appear as the second row for some matrix in $m$. In particular, there must be matrices in which $m_{3}$ belongs to $\mathrm{GF}\left(\mathrm{q}_{0}\right)$ but is not equal to zero. We have a contradiction which establishes the Lemma.

REMARK. A similar proof works for odd characteristic.

THEOREM 5.10. If $G_{1}$ and $H\left(\right.$ see (5.2)) are both non-solvable, then $G_{1}=\operatorname{SL}(2, q)$, $G_{2}=\operatorname{SL}\left(2, q_{2}\right)$ where $G F\left(q_{1}\right)$ and $G F\left(q_{2}\right)$ are subfields of $G F(q)$ such that $G F\left(q_{1}\right) \cap G F\left(q_{2}\right)=$ GF(2).

PROOF. By (3.4), (4.6) and (5.8) we have only to prove that $G F\left(q_{1}\right) \cap G F\left(q_{2}\right)$ cannot contain a subfield of order greater than 2. If $G F\left(q_{1}\right) \cap G F\left(q_{2}\right)=G F\left(q_{0}\right)$, where $\mathrm{q}_{0}>2$ we can apply Lemma (5.9) to get a contradiction.

\section{CONCLUSION}

We have tended to ignore subgroups of the translation complement which are both solvable and reducible or even solvable and irreducible but imprimitive. We have 
generally assumed that there is no normal 2-group. Here the subspace pointwise fixed by the normal 2 -group is invariant.

In all cases the induced group (the factor group modulo the subgroup fixing a minimal invariant subspace pointwise) is cyclic or dihedral in the solvable case. Thus the group theoretic structure is relatively uncomplicated. Yet "most" of the known planes of even order and dimension two are in this class. This includes most semi-field planes, generalized Hall planes and generalized André planes. We would conjecture that there are many more planes with an invariant component or Baer subplane, and we will not attempt to enumerate all of the possibilities.

Our investigations have led to two interesting possibilities for which we do not know whether or not examples exist. The first is described in (4.4); the second in $(5.10)$

\section{REFERENCES}

1. Dickson, L. E., Linear Groups, Dover, New York, 1958.

2. Foulser, D. A., Subplanes of parital spreads in translation planes, Bull. London Math. Soc. 4 (1978), 32-38.

3. Gorestein, Daniel, Finite Groups, Harper and Row, New York, 1968.

4. Hering, C., Zweifach transitive Permutationsgruppen, in denen 2 die maximale Anzahl von Fixpunkten von Involutionen ist, Mat. Z. 104 (1968), 150-174.

5. Hering, C., On subgroups with trivial normalizer intersection, J. Alg. 20 (1972), 622-629.

6. Hering, C., On shears of translation planes, Abh. Math. Sem. Hamb. $\underline{37}$ (1972), 258-268.

7. Hering, C., On projective planes of type VI, (unpublished).

8. Johnson, N. L., and Ostrom, T. G., Translation planes of characteristic two in which all involutions are Baer, J.Alg. 54 (1978), 291-315.

9. Kallaher, M. J., and Ostrom, T. G., Fixed point-free linear groups, rank three planes, and Bol quasifields, J. Alg. 18 (1971), 159-178.

10. Ostrom, T. G., Solvable linear groups on vector spaces whose dimension is the product of two primes, Aeg. Math. 18 (1978), 77-102

11. Ostrom, T. G., Collineations leaving a Desarguesian net invariant, Geom. Ded. 6 (1977), 109-120.

12. Ott, U., Eine neue Klasse indlicher Translationsebenen, Math. Z. 143 (1975), 181-185.

13. Passman, D. S., Permutation Groups, W. A. Benjamin, New York, 1968. 
14. Prohaska, 0., Endliche ableibare affinen Ebenen, Geom. Ded. 1 (1972), 6-17.

15. Suzuki, M., On a class of doubly transitive groups, Ann. Math. 75 (1962), 105-145.

16. Walter, J. H., The characterization of finite groups with abelian Sylo 2-groups, Ann. Math. 89 (second series) (1969), 404-514.

ADDED IN PROOF. Several people have called our attention to a paper by B. Mwene "On the subgroups of the group PSL $\left(4,2^{\mathrm{m}}\right) "$ J. Alg. 41 (1976), 79. The arguments in [8] could have been simplified if we had used Mwene's results. They probably also could be used in the present paper. 


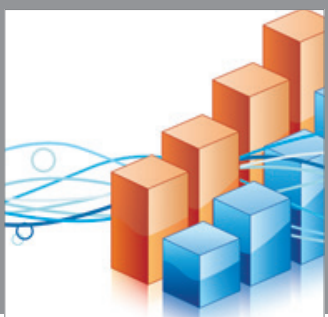

Advances in

Operations Research

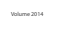

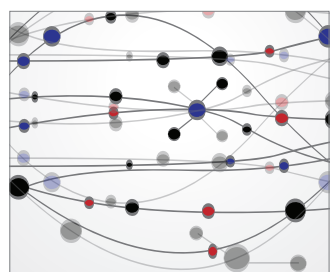

\section{The Scientific} World Journal
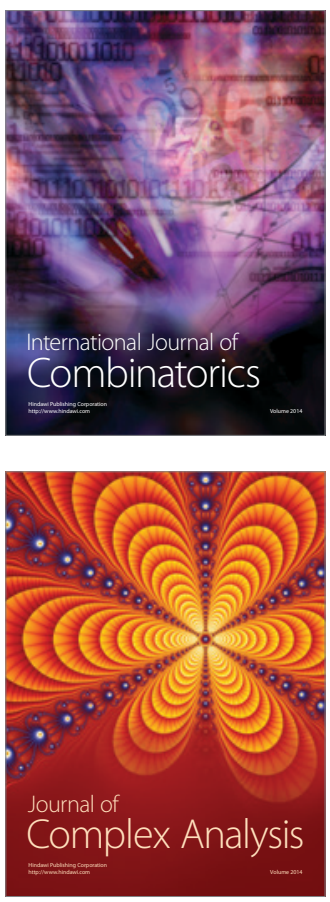

International Journal of

Mathematics and

Mathematical

Sciences
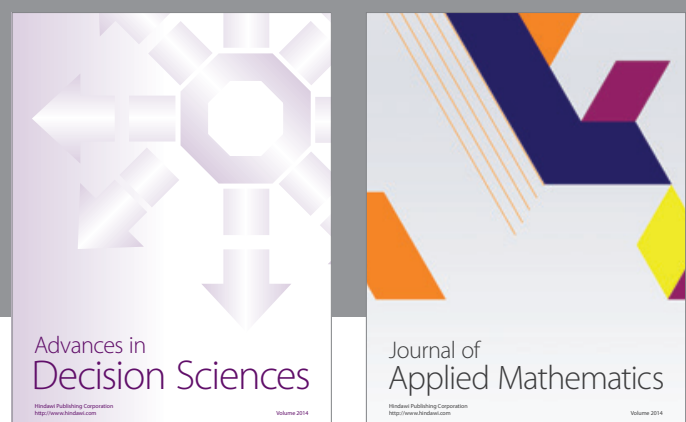

Journal of

Applied Mathematics
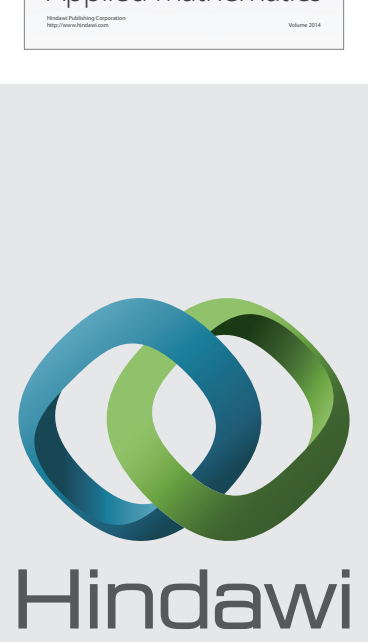

Submit your manuscripts at http://www.hindawi.com
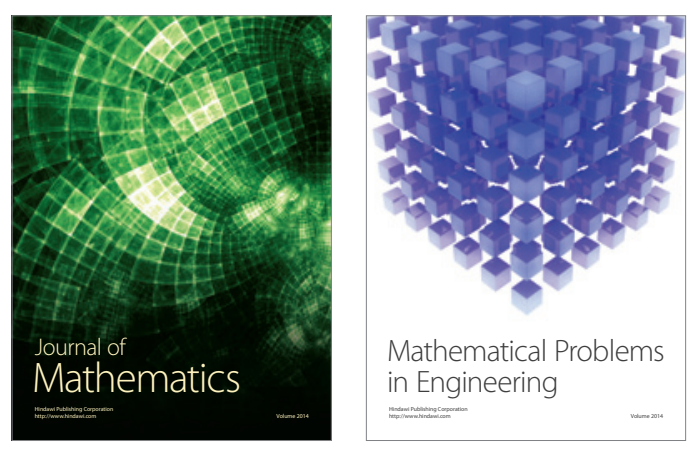

Mathematical Problems in Engineering
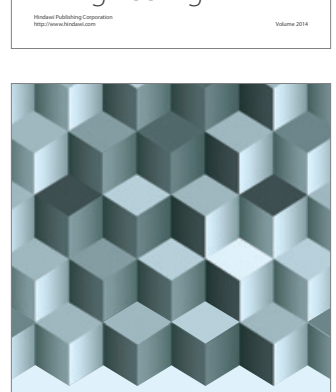

Journal of

Function Spaces
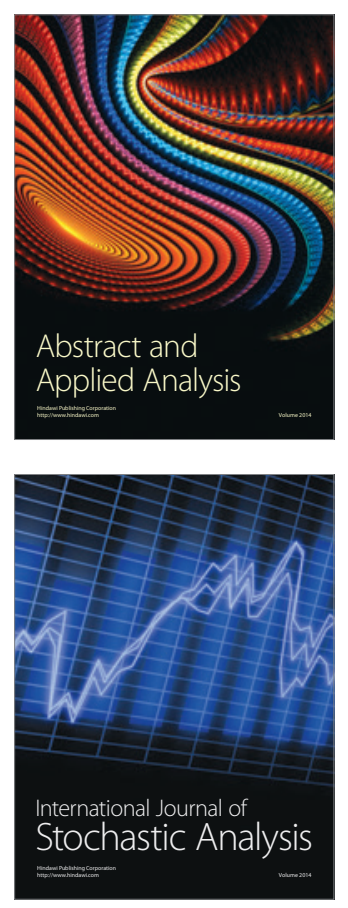

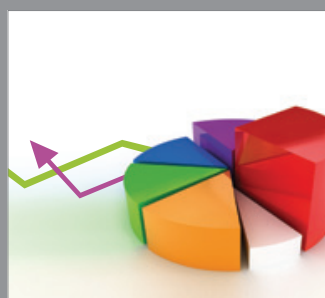

ournal of

Probability and Statistics

Promensencen
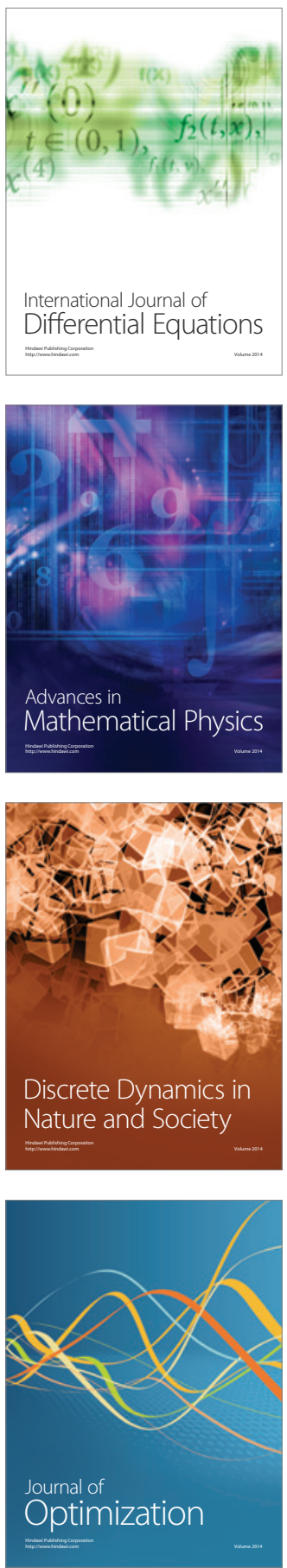